\title{
dspace.vutbr.cz
}

\section{Current-Mode Fractional-Order Electronically Controllable Integrator Design}

\author{
LANGHAMMER, L.; ŠOTNER, R.; DVOŘÁK, J.; SLÁDOK, O.; JEŘÁBEK, J.; BERTSIAS, P.
}

Proceedings of the 2020 IEEE International Conference on Electronics Circuits and Systems (ICECS)

elSBN: 978-1-7281-6044-3

DOl: https://doi.org/10.1109/ICECS49266.2020.9294923

Accepted manuscript

(C2020 IEEE. Personal use of this material is permitted. Permission from IEEE must be obtained for all other uses, in any current or future media, including reprinting/republishing this material for advertising or promotional purposes, creating new collective works, for resale or redistribution to servers or lists, or reuse of any copyrighted component of this work in other works. LANGHAMMER, L.; ŠOTNER, R.; DVOŘÁK, J.; SLÁDOK, O.; JEŘÁBEK, J.; BERTSIAS, P. "Current-Mode Fractional-Order Electronically Controllable Integrator Design", Proceedings of the 2020 IEEE International Conference on Electronics Circuits and Systems (ICECS), 2020. DOI: 10.1109/ICECS49266.2020.9294923. Final version is available at 


\section{Current-Mode Fractional-Order Electronically Controllable Integrator Design}

Lukas Langhammer, Roman Sotner, Jan Dvorak, Ondrej Sladok, Jan Jerabek

Faculty of Electrical Engineering and Communication Brno University of Technology

Technicka 12, 61600 Brno, Czech Republic

langhammer@feec.vutbr.cz

\author{
Panagiotis Bertsias \\ Department of Physics \\ University of Patras \\ 26504 Rio Patras, Greece
}

\begin{abstract}
This contribution presents a design of a currentmode fractional-order electronically controllable integrator which can be used as a building block for a design of fractionalorder (FO) circuits. The design is based on a $2^{\text {nd }}-$ order Followthe-Leader-Feedback topology which is suitably approximated to operate as an integrator of a fractional order. The topology is based on Operational Transconductance Amplifiers (OTAs), Adjustable Current Amplifiers (ACAs) and Current Follower (CF). The proposed structure offers the ability of the electronic control of its fractional order and also the electronic control of the frequency band. Simulations in Cadence IC6 (spectre) and more importantly experimental measurements were carried out to support the proposal. If wider bandwidth where the approximation is valid is required, a higher order structure must be used as also shown in this paper by utilization of a $4^{\text {th }}$ order FLF topology.
\end{abstract}

Keywords-current mode, electronic control, fractional order, fractional-order emulator, integrator

\section{INTRODUCTION}

One of the current research topics revolving around the analog circuit design is a topic of synthesis of fractional-order (FO) circuits and their utilization for modelling of systems with fractional-order characteristics, measurement of various samples in medicine and agriculture, or the implementation in the electrical engineering [1]. The FO systems can provide wider range of application in diverse technical areas due to their ability to provide characteristics in between integerorders in comparison to standard (integer-order) circuits.

The most typical approach to design of electrical circuits with FO characteristics is the employment of so-called Fractional-Order Elements (FOE). The FOE can be understood as a passive element with the slope of the attenuation of $20 \times \alpha \mathrm{dB} /$ decade, where parameter $\alpha$ stands for a real number in range $0<\alpha<1$. Similarly, the phase shift of such element is equal to $90 \times \alpha$ degrees. The FOE usually has the character of a FO capacitor (element with its character being between a capacitor and resistor). The impedance of such element is given as $Z_{\mathrm{C} \alpha}=1 / \boldsymbol{s}^{\alpha} C_{\alpha}$, where $\boldsymbol{s}^{\alpha}$ represents the FO Laplacian operator and $C_{\alpha}$ is a pseudo-capacitance with units of Farad/ $/ \mathrm{sec}^{1-\alpha}[2]$. There are three general ways how to obtain the FOE. The first technique is based on the fabrication of these elements [3-6] on various basis (electrochemical [3-4], resistive, capacitive and conductive layers on silicon [5] organic materials [6], for instance). The main disadvantages of this approach are the fact that the fabrication of these solutions is a complex and expensive process, commercial unavailability and the absence of the electronic control of the resulting order. The second method comprises the substitution of the FOC by RC ladder structures with suitably selected values [2], [7-9]. There are multiple topologies of the RC ladder structures such as Foster I, Foster II, Cauer I, and Cauer II, for instance [7]. The selected approximation of the FO Laplacian operator $\boldsymbol{s}^{\alpha}$ will determine the values of resistors and capacitors within the RC structure. The advantage is the requirement of only basic parts (standard resistors and capacitors). The drawback is the fact that we need specific values in comparison to the values of resistors and capacitors which are available only in fabrication series (in case of discrete implementation). Thus, the serial/parallels combinations are usually necessary. Moreover, the electronic control of the order is not available similarly to the previous approach (the values of the RC structure need to be recalculated for each value of FO). The last general way involves the use of a topology with electronically controllable active elements [10-15] to approximate the function of a FOE [10-12] or a FO building block (to behave as a FO integrator/differentiator) [13-15]. The disadvantage is higher complexity of the resulting structure and necessity of active elements. The advantage, on the other hand, is the presence of the electronic control of the order which can be easily set depending on the desired value of $\alpha$. Table I provides the comparative summary of mentioned FO emulators ([10-15]).

If considering valid operational bandwidth within range of $\pm 5^{\circ}$ (in case of the phase shift) from the ideal value, the solutions in [11], [13] and [14] provide less than two decades

\begin{tabular}{|c|c|c|c|c|c|c|c|}
\hline Ref No. & {$[10]$} & [11] & [12] & [13] & [14] & [15] & Fig. 2 \\
\hline $\begin{array}{c}\text { Number of active/passive } \\
\text { elements }\end{array}$ & $9 / 10$ & $9 / 2$ & $3 / 1^{\mathrm{a}}$ & $\mathrm{N} / \mathrm{A}^{\mathrm{b}}$ & $10 / 2$ & $14 / 5^{\mathrm{c}}$ & $\begin{array}{c}6 / 2 \\
10 / 4^{\mathrm{d}} \\
\end{array}$ \\
\hline Usable bandwidth & 2 decades & $<2$ decades & 4 decades & $<2$ decades & $<2$ decades & $<3$ decades & $\begin{array}{l}2 \text { decades/ } \\
<3 \text { decades }\end{array}$ \\
\hline El. BW shift control & Yes & Yes & $\mathrm{N} / \mathrm{A}^{\mathrm{e}}$ & $\mathrm{N} / \mathrm{A}^{\mathrm{e}}$ & $\mathrm{N} / \mathrm{A}^{\mathrm{e}}$ & $\mathrm{N} / \mathrm{A}^{\mathrm{e}}$ & Yes \\
\hline Simulations/Measurement & Yes/No & Yes/No & Yes/No & Yes/No & Yes/No & Yes/No & Yes/Yes \\
\hline
\end{tabular}

Note: ${ }^{2}$ Number of elements of one bilinear section which provides usable bandwidth less than one decade, ${ }^{\mathrm{b}} \mathrm{All}$ transistor-based topology, ${ }^{\mathrm{c}}$ The paper contains the solution based on other active elements as well. ${ }^{\mathrm{d}}$ Number of active/passive parts in case of the presented $4^{\text {th }}$-order FLF topology, ${ }^{\mathrm{e}}$ The feature not available or available but not investigated/presented

This article is based upon work from COST Action CA15225, a network supported by COST (European Cooperation in Science and Technology). Research described in this paper was financed by the Ministry of Education, Youth and Sports under grant LTC18022 of INTER-COST program. For the research, infrastructure of the SIX Center was used. 
where the approximation is valid. It is 2 decades for [10], $<3$ decades for [15] and it is 4 decades for [12] considering usage of 7 sections so the resulting number of active and passive elements is $21 / 7$. The design proposed in this paper offers valid bandwidth of $<2$ decades for $5 / 2$ parts or $<3$ decades for $9 / 5$ parts as discussed further. The proposed solution as the only one is also supported by experimental measurements.

\section{FRACTIONAL-ORDER INTEGRATOR DESIGN}

The proposed current-mode (having current input and current output) fractional-order integrator is based on the $2^{\text {nd }}$ order Follow-the-Leader-Feedback (FLF) topology with a FO approximation applied onto it so it behaves as a FO integrator (or differentiator if suitably calculated). The integrator was briefly introduced in [16] for the purpose of a demonstration of an electronically controllable FO of therein proposed structure, nonetheless, the integrator was not investigated closer. The topology itself is based on Operational Transconductance Amplifiers (OTAs), Adjustable Current Amplifiers (ACAs) and an auxiliary Current Follower (CF). Schematic symbols of the OTA, ACA and CF are presented in Fig. 1 a), b), c), respectively. The OTA element can be described by the relation $I_{\text {OUT }}= \pm g_{\mathrm{m}}\left(V_{\mathrm{IN}^{+}}-V_{\mathrm{IN}_{-}}\right)$, where $g_{\mathrm{m}}$ stands for the transconductance of this element. The behavior of the ACA can be expressed as $I_{\mathrm{OUT} \pm}= \pm B\left(I_{\mathrm{IN}}\right)$, where $B$ represents the current gain. The $\mathrm{CF}$ is described by $I_{\mathrm{OUT} \pm}= \pm I_{\mathrm{IN}}$. All mentioned active elements were simulated by the integrated building cells presented in [17]. The chip contains two multiplication units (CMOS and BJT), secondgeneration current conveyor CCII with four outputs, voltage differencing differential buffer (VDDB) and adjustable current amplifier. The OTA element has been created by the CMOS multiplication unit. Since the multiplication unit has only one output, the CCII (in form of a current follower) has been connected to this output in order to provide copies of the output current. The ACA has been created by the CMOS multiplication unit and one resistor (as shown in Fig. 1 d)) as the current amplifier in the chip has very limited dynamic range. The CCII was used to perform a function of the CF. The experimental measurements are done with a universal current conveyor (UCC) [18] substituting the OTA and CF elements and EL2082 [19] device to implement ACA element.

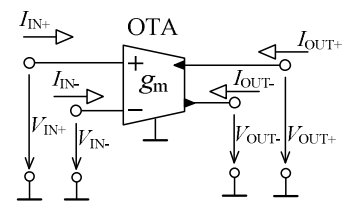

a)

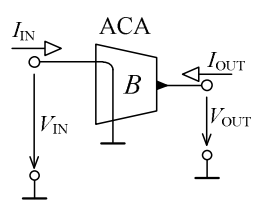

b)

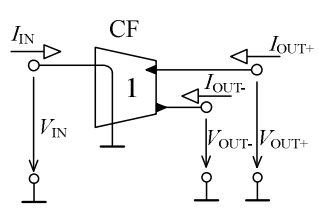

c)

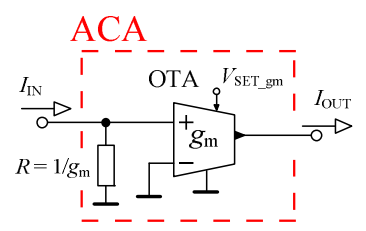

d)
Fig. 1. Schematic symbol of a) OTA, b) ACA, c) CF, d) implementation of ACA by a CMOS multiplication unit and one resistor

The $2^{\text {nd }}$-order FLF topology used for the approximation of the CM FO integrator is depicted in Fig. 2. It contains two OTAs, one CF, three ACAs (6 active elements in total) and two grounded capacitors. The number of active elements can be decreased to five as current gain $B_{2}$ is always equal to 1 (given by approximation) with a proper polarity of this branch in mind. The expected power consumption of the CMOSbased implementation is approximately $145 \mathrm{~mW}$.

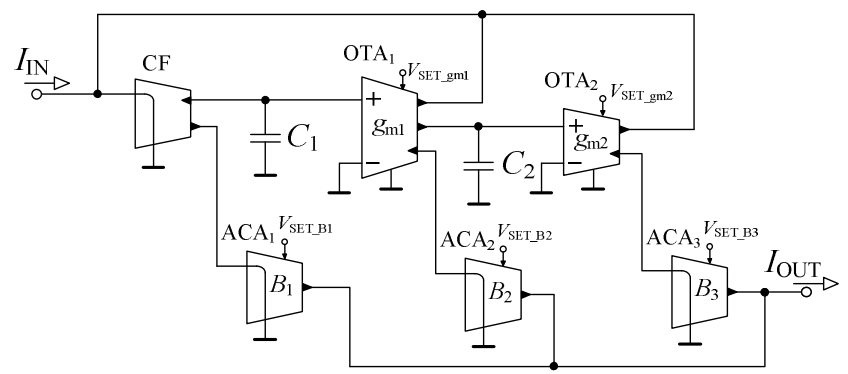

Fig. 2. $2^{\text {nd }}$-order FLF topology used for the approximation of a CM FO integrator

The transfer function of the structure in Fig. 2 is given by:

$$
K(\boldsymbol{s})=\frac{\boldsymbol{s}^{2} C_{1} C_{2} B_{1}+\boldsymbol{s} C_{2} g_{m 1} B_{2}+g_{m 1} g_{m 2} B_{3}}{\boldsymbol{s}^{2} C_{1} C_{2}+\boldsymbol{s} C_{2} g_{m 1}+g_{m 1} g_{m 2}} .
$$

The second-order approximation of $\boldsymbol{s}^{\alpha}$ is expressed as:

$$
\boldsymbol{s}^{\alpha} \cong \frac{a_{0} \boldsymbol{s}^{2}+a_{1} \boldsymbol{s}+a_{2}}{a_{2} \boldsymbol{s}^{2}+a_{1} \boldsymbol{s}+a_{0}} .
$$

The values of $a_{0}, a_{1}, a_{2}$ (when using a second-order approximation provided in [18]) are being given as $a_{0}=\alpha^{2}+3 \alpha$ $+2, a_{1}=8-2 \alpha^{2}, a_{2}=\alpha^{2}-3 \alpha+2$ (obtained by Continued fraction expansion (CFE) method). Thus, the general transfer function which approximates the $2^{\text {nd }}$-order FLF topology in order to behave as a FO integrator is given as:

$$
K(s)=\frac{\left(\frac{\alpha^{2}-3 \alpha+2}{\alpha^{2}+3 \alpha+2}\right) s^{2}+\frac{1}{\tau}\left(\frac{8-2 \alpha^{2}}{\alpha^{2}+3 \alpha+2}\right) s+\frac{1}{\tau^{2}}}{s^{2}+\frac{1}{\tau}\left(\frac{8-2 \alpha^{2}}{\alpha^{2}+3 \alpha+2}\right) s+\frac{1}{\tau^{2}}\left(\frac{\alpha^{2}-3 \alpha+2}{\alpha^{2}+3 \alpha+2}\right)} .
$$

The values of each variable in (1), for selected value of $\alpha$ and chosen operational frequency, can be determined when comparing (1) with (3).

\section{SIMULATION AND EXPERIMENTAL RESULtS}

In order to support the proposed design, simulations in Cadence IC6 (spectre) (using CMOS I3T25 $0.35 \mu \mathrm{m} \mathrm{ON}$ Semiconductor technology) and experimental measurements were carried out. The dependency of the transconductance of used multiplication unit on the driving DC voltage is given as $g_{\mathrm{m}} \approx\left(2 \times V_{\text {SET_gm }}\right) / 1000$. Simple $\mathrm{V}-\mathrm{I} / \mathrm{I}-\mathrm{V}$ convertors together with network analyzer Agilent 4395A were used for the measurement. The central operational frequency $f_{\mathrm{C}}$ of the bandwidth where the approximation is valid has been chosen to be $50 \mathrm{kHz}$. The values of capacitors are as follows $C_{1}=1$ $\mathrm{nF}$ and $C_{2}=10 \mathrm{nF}$. The values of transconductances $g_{\mathrm{m} 1}$ and $g_{\mathrm{m} 2}$ and current gains $B_{1}, B_{2}, B_{3}$ are then calculated based on the selected operational frequency and chosen approximation.

The electronic control of FO of the proposed integrator is demonstrated in Fig. 3 for the simulation results represented by black dashed lines and the experimental results denoted by colored lines. The results are provided for $\alpha$ equal to 0.1 to 0.7 with step of 0.1 . Specific values of transconductances and current gains in dependence on $\alpha$ are provided in Table II. As it is evident from the table, higher values of $\alpha$ require 
values of current gains which are more difficult to obtain (either too low $\left(B_{1}\right)$ or too high $\left(B_{3}\right)$ ). Thus, the proposed circuit is more suitable for lower values of $\alpha$. From the graphs, it can be seen that the approximation of fractional order is valid for approximately two decades. The usable bandwidth is also limited by the frequency limitations (effect of parasitic characteristics) of used active elements as observable at frequencies above $3 \mathrm{MHz}$. The experimental values (in case of the magnitude) are slightly (about $3 \mathrm{~dB}$ ) higher than the simulation results, nonetheless, both results support the intended function and design correctness.

TABLE II. VALUES OF INDIVIDUAL PARAMETERS OF THE INTEGRATOR IN DEPENDENCE ON VALUES OF ALPHA

\begin{tabular}{|c|c|c|c|c|c|c|c|}
\hline $\boldsymbol{\alpha}[-]$ & $\mathbf{0 . 1}$ & $\mathbf{0 . 2}$ & $\mathbf{0 . 3}$ & $\mathbf{0 . 4}$ & $\mathbf{0 . 5}$ & $\mathbf{0 . 6}$ & $\mathbf{0 . 7}$ \\
\hline $\boldsymbol{g}_{\mathrm{m} 1}[\boldsymbol{\mu S}]$ & 1090 & 942 & 822 & 718 & 628 & 550 & 480 \\
\hline $\boldsymbol{g}_{\mathrm{m} 2}[\boldsymbol{\mu S}]$ & 673 & 571 & 478 & 393 & 314 & 242 & 175 \\
\hline $\mathbf{B} 1[-]$ & 0.74 & 0.545 & 0.398 & 0.286 & 0.2 & 0.135 & 0.085 \\
\hline $\mathbf{B} 2[-]$ & & & 1 & & \\
\hline B3 [-] & 1.35 & 1.833 & 2.51 & 3.5 & 5 & 7.43 & 11.77 \\
\hline
\end{tabular}

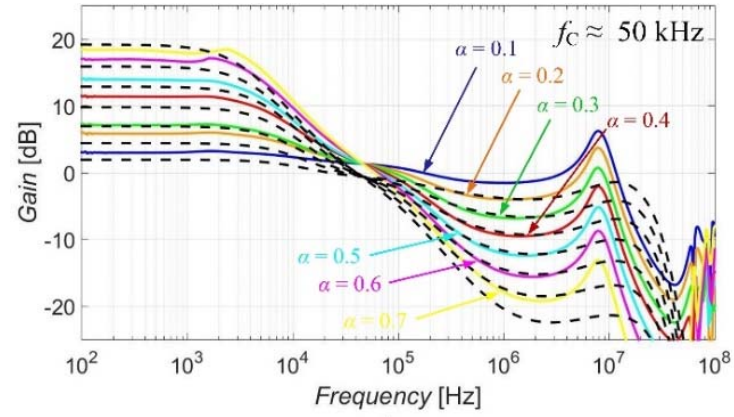

a)

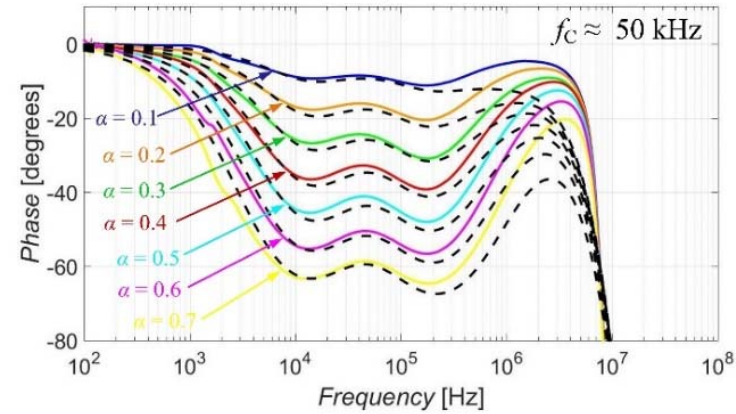

b)

Fig. 3. Electronic control of the order of proposed FO integrator (experimental measurment (colored lines), simulations (black dashed lines)): a) magnitude characteristics, b) phase characteristics

The other beneficial electronic control is the control of the frequency of the operational bandwidth where the approximation is valid. The control id achieved through the change of transconductances $\left(g_{\mathrm{m} 1}\right.$ and $\left.g_{\mathrm{m} 2}\right)$ as long as the ratio between them stays unchanged. That way we can shift the bandwidth to desired frequencies. Fig. 4 demonstrates the frequency shift of the operational bandwidth for three different settings of transconductances (provided in the Table III) where the theoretical (center) frequency $f_{\mathrm{C}}$ is approximately equal to $25 \mathrm{kHz}, 50 \mathrm{kHz}$ and $100 \mathrm{kHz}$. The demonstration is done for $\alpha=0.5$, thus the setting of current gains is the same as for $\alpha=0.5$ in Table II for all three cases in Fig. 4. Lower upper DC gain of the $25 \mathrm{kHz}$ characteristic is most likely caused by limited linear behavior based on the values of transconductances.

TABLE III. VALUES OF TRANSCONDUCTANCES (FOR ALPHA $=0.5)$ IN DEPENDENCE ON THE CONTROL OF THE FREQUENCY BAND

\begin{tabular}{|c|c|c|c|}
\hline Theoretical frequency $\boldsymbol{f}_{\boldsymbol{C}}$ & $\mathbf{2 5} \mathbf{~ k H z}$ & $\mathbf{5 0} \mathbf{~ k H z}$ & $\mathbf{1 0 0} \mathbf{~} H \mathbf{z}$ \\
\hline $\boldsymbol{g}_{\mathrm{m} 1}[\boldsymbol{\mu S}]$ & 314 & 628 & 1256 \\
\hline $\boldsymbol{g}_{\mathrm{m} 2}[\boldsymbol{\mu S}]$ & 157 & 314 & 628 \\
\hline
\end{tabular}

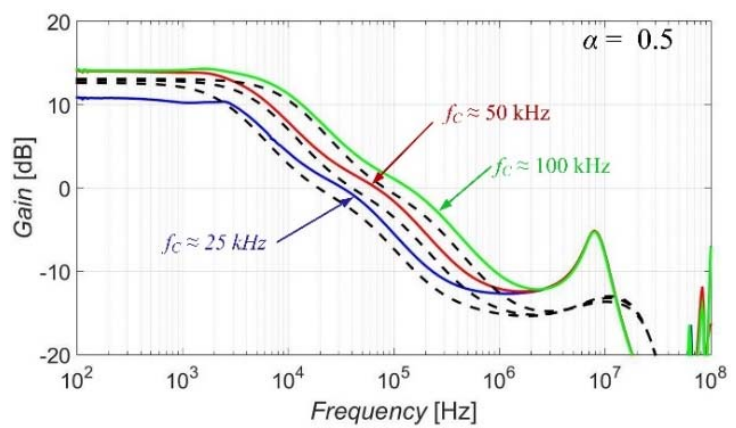

a)

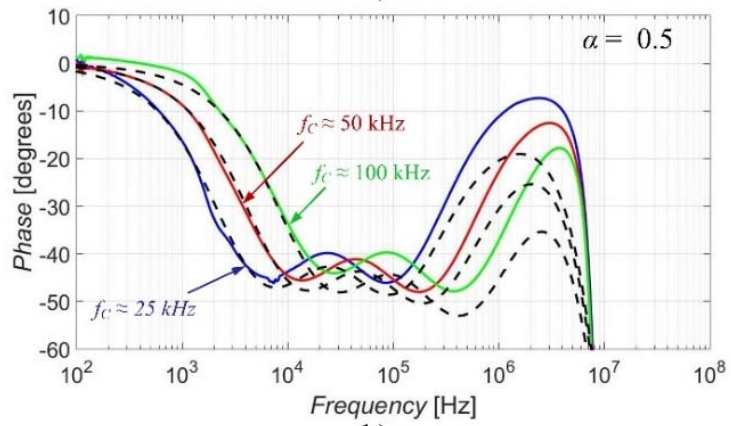

b)

Fig. 4. Electronic control of the frequency band of proposed FO integrator (experimental measurment (colored lines), simulations (black dashed lines)): a) magnitude characteristics, b) phase characteristics

In order to provide wider operational bandwidth, the analogous $4^{\text {th }}$-order FLF topology (Fig. 5) has been designed. It consists of one CF, four OTAs, five ACAs and four grounded capacitors. Since the value of current gain $B_{3}$ is always 1 , the $\mathrm{ACA}_{3}$ can be omitted decreasing the number of active elements by one ( 9 in total). The $4^{\text {th }}$-order approximation provided in [15] has been used. When choosing the operational frequency being approximately equal to $50 \mathrm{kHz}$ and $\alpha=0.5, C_{1}=100 \mathrm{pF}, C_{2}=C_{3}=1 \mathrm{nF}, C_{4}=10 \mathrm{nF}$, the values of remaining parameters were calculated accordingly: $g_{\mathrm{m} 1}=293 \mu \mathrm{S}, g_{\mathrm{m} 2}=471 \mu \mathrm{S}, g_{\mathrm{m} 3}=898 \mu \mathrm{S}$, $g_{\mathrm{m} 4}=87 \mu \mathrm{S}, B_{1}=0.11 B_{2}=0.43 B_{3}=1, B_{4}=2.33 B_{5}=9$. The values of transconductances and current gains were obtained in similar manner as in case of the topology from Fig. 2 by comparison of the transfer function of the proposed structure and the relation of the $4^{\text {th }}$-order FO approximation. The transfer function of the structure in Fig. 5 is given as $K(\boldsymbol{s})=N(\boldsymbol{s}) / D(\boldsymbol{s})$, where:

$$
\begin{aligned}
& N(\boldsymbol{s})=\boldsymbol{s}^{4} C_{1} C_{2} C_{3} C_{4} B_{1}+\boldsymbol{s}^{3} C_{2} C_{3} C_{4} g_{m 1} B_{2}+ \\
& +\boldsymbol{s}^{2} C_{3} C_{4} g_{m 1} g_{m 2} B_{3}+s C_{4} g_{m 1} g_{m 2} g_{m 3} B_{4}+, \\
& g_{m 1} g_{m 2} g_{m 3} g_{m 4} B_{5}
\end{aligned}
$$




$$
\begin{aligned}
& D(s)=s^{4} C_{1} C_{2} C_{3} C_{4}+s^{3} C_{2} C_{3} C_{4} g_{m 1}+ \\
& +s^{2} C_{3} C_{4} g_{m 1} g_{m 2}+s C_{4} g_{m 1} g_{m 2} g_{m 3}+ \\
& g_{m 1} g_{m 2} g_{m 3} g_{m 4}
\end{aligned}
$$

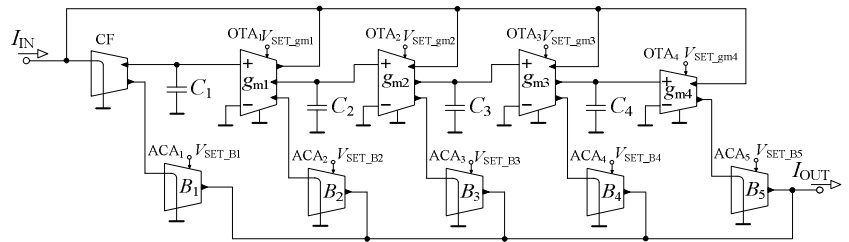

Fig. 5. $4^{\text {th }}-$ order FLF topology used for the approximation of a CM FO integrator

The comparison of the simulation results (colored lines) and theoretical expectations (black dashed lines) of the integrator from Fig. 2 (blue lines) and the integrator from Fig. 5 (red lines) is presented in Fig. 6. It can be seen that the integrator from Fig. 5 can provide wider bandwidth where the approximation is valid (about three decades) in comparison to the integrator from Fig. 2 (about two decades). It can provide wider bandwidth with the same amount of active elements (10(9) in this case) in comparison to solutions in Table I. It can be seen that the usable bandwidth is depending on the order of the used approximation. Considering $5^{\circ}$ phase error for the case when $\alpha=0.5$, the bandwidth is given as approximately $f_{\mathrm{c}} / 10$ and $f_{\mathrm{c}} \cdot 10$ for order of used approximation $n=2$ and $f_{\mathrm{c}} / 25$ and $f_{\mathrm{c}} \cdot 10$ for $n=4$.

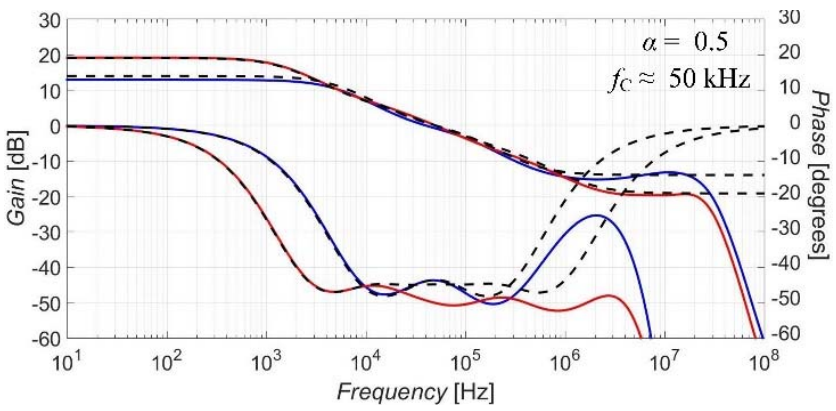

Fig. 6. Comparison of the simulation (colored lines) and theoretical (black dashed lines) results of the integrator from Fig. 2 (blue lines) and the integrator from Fig. 5 (red lines)

\section{CONCLUSION}

Cadence IC6 (spectre) simulations and experimental measurements were carried out to support the design. The electronic controls of the FO and frequency band were proven to function as intended (see Figs. 3 and 4). The design requires five active elements and two passive elements in case of the $2^{\text {nd }}-$ order topology (with usable bandwidth of about 2 decades) and nine active and four passive elements in case of the $4^{\text {th }}$-order topology (with usable bandwidth of about 3 decades) which is less active elements needed in comparison to other proposed integrators while providing same bandwidth range. The proposed integrator can also function as a FO differentiator when calculated accordingly.

\section{REFERENCES}

[1] A. S. Elwakil, "Fractional-order circuits and systems: An emerging interdisciplinary research area," IEEE Circuits and Systems Magazine, Vol. 10, No. 4, 2010, pp. 40-50.
[2] T. J. Freeborn, "A Survey of Fractional-Order Circuit Models for Biology and Biomedicine," IEEE Journal on Emerging and Selected Topics in Circuits and Systems, Vol. 3, No. 3, 2013, pp. 416-424.

[3] C. L. Alexander, B. Tribollet M. E.Orazem, "Contribution of Surface Distributions to Constant-Phase-Element (CPE) Behavior:1.Influence of Roughness," Electrochimica Acta, Vol. 173, 2015, pp. 416-424.

[4] A. Adhikary, M. Khanra, S. Sen, K. Biswas, "Realization of a Carbon Nanotube Based Electrochemical Fractor," In Proc. 2015 IEEE International Symposium on Circuits and Systems (ISCAS), Lisbon, Portugal, 2015, pp. 2329-2332.

[5] P. Ushakov, A. Shadrin, D. Kubanek, J. Koton, "Passive FractionalOrder Components Based On Resistive-Capacitive Circuits With Distributed Parameters," In Proc. 39th International Conference on Telecommunications and Signal Processing (TSP), Vienna, Austria, 2016, pp. 638-642.

[6] S. Amand, M. Musiani, M. E. Orazem, N. Pébère, B. Tribollet, V. Vivier, "Constant-phase-element behavior caused by inhomogeneous water uptake in anti-corrosion coatings," Electrochimica Acta, Vol. 87, 2013, pp. 693-700.

[7] G. Tsirimokou, "A systematic procedure for deriving RC networks of fractional-order elements emulators using MATLAB," Int. Journal of Electronics and Communications (AEÜ), Vol. 78, 2017, pp. 7-14.

[8] J. Valsa, J. Vlach, "RC models of a constant phase element, international journal of circuit theory and applications," Vol. 41, 2013, pp. 59-67.

[9] O. Domansky, R. Sotner, L. Langhammer, J. Jerabek, C. Psychalinos, G. Tsirimokou, "Practical Design of RC Approximants of Constant Phase Elements and Their Implementation in Fractional-Order PID Regulators Using CMOS Voltage Differencing Current Conveyors," Circuits, Systems, and Sign. Processing, Vol. 38, 2019, pp. 1520-1546.

[10] J. Dvorak, D. Kubanek, N. Herencsar, A. Kartci, P. Bertsias, "Electronically Adjustable Emulator of the Fractional Fractional-Order Capacitor," Elektronika ir Elektrotechnika, Vol. 25, No. 6, 2019, pp. 28-34.

[11] G. Tsirimokou, C. Psychalinos, A. S. Elwakil, "Emulation of a constant phase element using operational transconductance amplifiers," Analog Integrated Circuits and Signal Processing, Vol. 85, 2015, pp. 413-423.

[12] R. Sotner, J. Jerabek, J. Petrzela, T. Dostal, "Simple Approach for Synthesis of Fractional-Order Grounded Immittances Based on OTAs," In Proc. 39th International Conference on Telecommunications and Signal Processing (TSP), Vienna, Austria, 2016, pp. 563-568.

[13] G. Tsirimokou, C. Psychalinos, "Ultra-low voltage fractional-order circuits using current mirrors," International Journal of Circuit Theory and Applications, Vol. 44, 2016, pp. 109-126.

[14] M. R. Dar, N. A. Kant, F. A. Khanday, "Realization of Fractional-Order Double-Scroll Chaotic System Using Operational Transconductance Amplifier (OTA)," Journal of Circuits, Systems, and Computers, Vol. 27, No. 1, 2018, pp. 1-15.

[15] G. Tsirimokou, A. Kartci, J. Koton, N. Herencsar, C. Psychalinos, "Comparative Study of Discrete Component Realizations of Fractional-Order Capacitor and Inductor Active Emulators," Journal of Circuits, Systems, and Computers, Vol. 27, No. 11, 2018, pp. 1-26.

[16] L. Langhammer, J. Dvorak, R. Sotner, J. Jerabek, P. Bertsias, "Reconnection-less reconfigurable low-pass filtering topology suitable for higher-order fractional-order design," Journal of Advanced Research, 2020, accepted for publication.

[17] R. Sotner, J. Jerabek, L. Polak, R. Prokop, V. Kledrowetz, "Integrated Building Cells for a Simple Modular Design of Electronic Circuits with Reduced External Complexity: Performance, Active Element Assembly, and an Application Example," Electronics, Vol. 8, Iss. 5, 2019, pp. $1-26$.

[18] UCC-N1B - Universal Current Conveyor (UCC) and SecondGeneration Current Conveyor (CCII+/-) (datasheet), Brno University of Technology, Czech Republic, 2010, accessible on http://www.utko.feec.vutbr.cz/ koton/soubory/UCC_N1B_Rev0.pdf

[19] Intersil (Elantec), EL2082 CN Current-mode multiplier (datasheet), 1996 , accessible on http://pdf.datasheetcatalog.com/datasheet/elantec/EL2082CN.pdf.

[20] T. J. Freeborn, B. Maundy, A. S. Elwakil, "Field programmable analogue array implementation of fractional step filters," IET Circuits, Devices \& Systems, Vol. 4 , Iss. 6, 2010, pp. 514-524. 